\title{
The Importance of Measurement Data Spacing
}

The Physics Teacher 53, 356 (2015); https:// doi.org/10.1119/1.4928351

T. M. Seixas and M. A. Salgueiro da Silva

View Affiliations

的

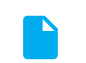

느

9<smiles>[CH]1CC1</smiles> 


\section{ABSTRACT}

When conducting experiments involving the measurement of physically related quantities, choosing an appropriate spacing for the experimental independent variable is a crucial procedure whose consequences may go beyond data graphical visualization. This is particularly true if the measured quantities are nonlinearly related and experimental errors are nonuniform. In this work, we show that measurement procedures should provide a higher density of data points in the range where experimental errors are larger, to more effectively average out random errors and thus mitigate accuracy errors in parameter determination. Knowing the effective experimental independent variable helps in defining an adequate data spacing.

\section{SELECT YOUR ACCESS}

\section{INDIVIDUAL ACCESS}

If you have an individual subscription, a subscription provided by one of AIP's Member Societies, have claimed access to a Conference Proceeding, or have made an individual purchase, sign in below.

Username:

Password 


\section{INSTITUTIONAL ACCESS}

Log in via Open Athens

Log in via Shibboleth

\section{PURCHASE}

Standard PPV for $\$ 30.00$

ADD TO CART

\section{Resources}

\section{AUTHOR}

LIBRARIAN

ADVERTISER

\section{General Information}

ABOUT

CONTACT

HELP

PRIVACY POLICY 
TERMS OF USE

FOLLOW AIP PUBLISHING:

$f y$ in

Website (c) 2018 AIP Publishing LLC. Article copyright remains as specified within the article. 